\title{
Correction: Prioritization of Free-Text Clinical Documents: A Novel Use of a Bayesian Classifier
}

\author{
Mark Singh $^{1^{*}}$, BE(Elec), MD; Akansh Murthy ${ }^{2^{*}}, \mathrm{BS} ;$ Shridhar Singh ${ }^{3^{*}}$ \\ ${ }^{1}$ Carnegie Mellon University, University of Massachusetts Medical School, Braintree, MA, United States \\ ${ }^{2}$ Massachusetts Institute of Technology, Cambridge, MA, United States \\ ${ }^{3}$ Carnegie Mellon University, Pittsburgh, PA, United States \\ ${ }^{*}$ all authors contributed equally
}

Corresponding Author:

Akansh Murthy, BS

Massachusetts Institute of Technology

77 Mass Ave

Cambridge, MA, 02139

United States

Phone: 16172531000

Email: ambshun@mit.edu

\section{Related Article:}

Correction of: https://medinform.jmir.org/2015/2/e17/

(JMIR Med Inform 2020;8(6):e21379) doi: 10.2196/21379

In "Prioritization of Free-Text Clinical Documents: A Novel Use of a Bayesian Classifier" (JMIR Med Inform 2015;3(2):e17) the authors noted an error in corresponding author's details.

The corrected information is:

Akansh Murthy, BS

Massachusetts Institute of Technology

77 Mass Ave

Cambridge, MA, 02139

\section{United States}

Phone: 16172531000

Email:ambshun@mit.edu

The correction will appear in the online version of the paper on the JMIR Publications website on June 23, 2020, together with the publication of this correction notice. Because this was made after submission to PubMed, PubMed Central, and other full-text repositories, the corrected article has also been resubmitted to those repositories

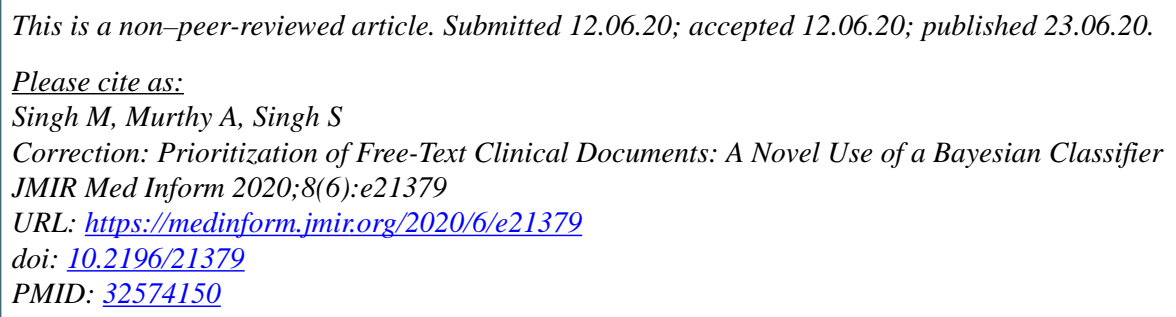

CMark Singh, Akansh Murthy, Shridhar Singh. Originally published in JMIR Medical Informatics (http://medinform.jmir.org), 23.06.2020. This is an open-access article distributed under the terms of the Creative Commons Attribution License (https://creativecommons.org/licenses/by/4.0/), which permits unrestricted use, distribution, and reproduction in any medium, provided the original work, first published in JMIR Medical Informatics, is properly cited. The complete bibliographic information, a link to the original publication on http://medinform.jmir.org/, as well as this copyright and license information must be included. 